\title{
Interspecific ovarian transplantations in Drosophila: vitellogenin uptake as an index of evolutionary relatedness
}

\author{
Klea Lamnissou* and \\ E. Zouros $\dagger$
}

\author{
* Department of Biochemistry, Cell and Molecular \\ Biology and Genetics, University of Athens, Athens, \\ Greece. \\ †Department of Biology, The University of Crete, \\ Iraklion, Crete, Greece, and \\ Department of Biology, Dalhousie University, \\ Halifax, Nova Scotia, Canada, B3H 4J1.
}

Evolutionary divergence is measured either by comparing homologous characters in related species or by assessing the compatibility of genes, cells or tissues from different species to sustain normal development and function. Here we examine vitellogenin accumulation in interspecifically transplanted ovaries as a measure of evolutionary divergence of the second kind. We report on the results from about $\mathbf{4 0 0}$ ovary transplantations involving 27 species from four different species groups of Drosophila. With the exception of one species, no vitellogenin accumulation was observed in inter-group transplantations, but accumulation occurred at varying degrees in intra-group transplantations. This degree is in good agreement with intra-group phylogenetic relationship established on other criteria and it can, therefore, be a useful complementary index of relative evolutionary divergence among members of the same species group.

\section{INTRODUCTION}

Most studies of evolutionary distance of related species have used characters whose degree of divergence is being studied without reference to (or in ignorance of) the character's "role" in the development or function of the organism. Chromosomal inversions (e.g., Wasserman, 1960), allozymes (e.g. Zouros, 1973), and variation scored at the DNA level (e.g., Coyne and Kreitman, 1986) are among the most typical examples of this type of character.

A second category of character that has also been used for the inference of evolutionary relatedness, relates to the degree to which combinations of genes, cells, or tissues from different organisms are compatible with the development of a normal phenotype. Because they are scored through the manifestation of their role, these characters cannot be treated as neutral to the forces of natural selection nor can they be assumed to have time-dependent rates of evolution. Also, they normally have two-state (yes-or-no) discontinuous distributions, in contrast to the continuous distributions of the characters of the first category. Given these differences, an important question is how concordant are the phylogenetic relationships derived from the two classes of character.
In Drosophila, the compatibility between genomes from different species has been studied mostly through hybridization which, however, can be applied only to very closely related species. The simple technique of transplanting immature ovaries from newly hatchet females of one species (the donor species) into females of another species (the host species) is free of this limitation. In such transplantations the scored quantity is oocyte development in the guest (donor) ovary. Previous studies using this assay have largely concentrated on developmental aspects of vitellogenin production and uptake or on the general question of ovary growth in interspecific transplantations (Kambysellis, 1970; Srdic et al., 1978). Here we explore the possibility for the use of this assay as an index of phylogenetic relationship.

\section{MATERIALS AND METHODS}

The following Drosophila species were used. Melanogaster subgroup: melanogaster, simulans, mauritiana, yakuba, erecta, teissieri, orena; montium subgroup: auraria, serrata, jumbulina; willistoni group: willistoni, tropicalis, paulistorum, equinoxialis; repleta group: mojavensis, 
arizonensis, mulleri, hydei, eohydei, mercatorum, repleta, peninsularis; virilis group: virilis, americana americana, montana, borealis.

Transplantations of ovaries were performed as described before (King and Bodenstein, 1965; Postlethwait and Handler, 1978). Ovaries were removed from the donor species and transplanted into the host when both females were 2 hours old. Depending on the host species, host females were dissected 3 to 5 days after the operation, and the stage of oocyte development was scored in the guest ovary as well as in the two native ovaries. Transplanted ovaries were easily recognized from the native ones because they fail to attach to the host's oviducts. Oocyte development was measured using King's (1970) scale which recognizes fourteen developmental stages. Of these, stages 1 to 7 are pre-vitellogenic and 8 to 14 are vitellogenic. For each ovary the maximum developmental stage (MDS) reached by one or more oocytes was scored. For any donor/host combination we have performed several transplantations. For an overall measure of oocyte development for a given combination, we used the mean of MDS, as done previously by Kambysellis (1970). We will refer to this mean as the index of maximum developmental stage (IMDS). The index is good only as an indication of general performance of transplanted ovaries, but it cannot be used for statistical inference. For comparing the results from transplantations involving two different donor/host combinations we used the Wilcoxon two-sample ranking non-parametric test (Brownlee, 1965).

An important condition of our experiments was that transplanted ovaries remained live within the host environment and did not lose their innate capacity for vitellogenin uptake if found in a permissible environment. The first condition was checked by observing, upon dissection of the host female, whether the transplanted ovary has grown at all. Atrophied, disintegrated or dead ovaries were not included in the data set. The second condition was checked by performing several "double transplantations" consisting in removing ovaries that have failed to develop to post-vitellogenic stages from their hosts (normally belonging to a different species group) and retransplanting them in females of their own species. Finally, to control for the possibility that a foreign ovary failed to develop not because of incompatibility with the host, but rather because of inability of the particular female host to sustain ovary development, we examined in each case the foreign as well as the two native ovaries.

\section{RESULTS}

Five types of "double transplantations" were performed: melanogaster/virilis/melanogaster, serrata/melanogaster/serrata, willistoni/melanogaster/willistoni, virilis / hydei/virilis and equinoxialis/mercatorum/equinoxialis. In each triad the first species was the original donor of the ovary, the second species was the first host and the third species was the second host. Each combination was repeated several times. In all cases the ovaries failed to develop while in the heterospecific host, but developed fully when transplanted in the conspecific host. These data illustrate the ability of ovaries to retain the potential for vitellogenin uptake when found in the appropriate environment, as was earlier observed (Srdic et al., 1978).

\section{The melanogaster species group}

The taxonomy, biogeography, cytology, and crosshybridization of this species group have been reviewed by Lemeunier et al. (1986). We have used 11 species from this group. Of these seven belonged to the melanogaster subgroup, three to the montium subgroup and one to the ananassae subgroup. Both species complexes (the melanogaster complex and the yakuba complex) of the melanogaster subgroup were represented among these species. The three montium species also belonged to different species complexes. The results are shown in table 1(a). The Wilcoxon two-sample rank test is significant for the erecta/melanogaster versus the mauritiana/melanogaster comparison $(u=1 \cdot 81$, $P=0.035)$ where the numbers of tested ovaries were relatively large, but it is not significant for other pair-wise comparisons (the next two most different comparisons erecta/melanogaster versus melanogaster/melanogaster and erecta/melanogaster versus simulans/melanogaster both yield $u=1.58, P=0.057)$. The mean within-complex index ( simulans or mauritiana ovaries transplanted into melanogaster) is different from the acrosscomplex index (yakuba, teissieri, orena or erecta ovaries transplanted into melanogaster). The significance of the difference of the two means can be tested by pooling all within-complex scores into one group and all across-complex scores into another (this pooling is a permissible a priori operation given the well recognized subdivision of the melanogaster subgroup into two complexes). The Wilcoxon test for the two sets gives $u=2 \cdot 53$, $P=0.006$. None of the three pair-wise comparisons in the montium subgroup are statistically different, nor is the intraspecific set 
Table 1 Results from ovary transplantations among species of the melanogaster (a), the willistoni (b), the repleta (c) and the virilis (d) groups of Drosophila. The first species is the donor and the second is the host. $n=$ number of successful transplantations, IMDS $=$ the mean of maximum developmental stage, $\mathrm{D}=$ allozyme distance. Numbers in parenthesis are the maximum developmental stages of ovaries that produced no oocyte of stage 14. Taxonomic designations (in nested order) refer to the donor species. Results from statistical tests are given in the text

\begin{tabular}{|c|c|c|c|}
\hline & $n$ & IMDS & $\mathrm{D}$ \\
\hline \multicolumn{4}{|l|}{$\begin{array}{l}\text { (a) Melanogaster group } \\
\text { subgroup: melanogaster } \\
\text { complex: melanogaster }\end{array}$} \\
\hline species: melanogaster/melanogaster & 7 & 14 & - \\
\hline species: simulans/melanogaster & 6 & 14 & 0.325 \\
\hline $\begin{array}{l}\text { species: mauritiana/melanogaster } \\
\text { complex: yakuba }\end{array}$ & 15 & $13 \cdot 73(10)$ & $0 \cdot 325$ \\
\hline species: yakuba/melanogaster & 15 & $13 \cdot 13(10,2 \times 11,12,13)$ & 1.099 \\
\hline species: teisseiri/melanogaster & 6 & $13 \cdot 00(8)$ & $0 \cdot 811$ \\
\hline species: orena/melanogaster & 9 & $12 \cdot 67(9,10,11)$ & - \\
\hline $\begin{array}{l}\text { species: erecta/melanogaster } \\
\text { subgroup: montium } \\
\text { complex: auraria }\end{array}$ & 22 & $12 \cdot 41(3 \times 8,9,10,11,2 \times 12,13)$ & 1.099 \\
\hline species: auraria/auraria & 5 & 14 & - \\
\hline $\begin{array}{l}\text { species: auraria/melanogaster } \\
\text { complex: serrata }\end{array}$ & 7 & $<7$ & - \\
\hline $\begin{array}{l}\text { species: serrata/auraria } \\
\text { complex: undefined }\end{array}$ & 13 & $12.31(8,9,2 \times 10,11)$ & 0.73 \\
\hline $\begin{array}{l}\text { species: jambulina/auraria } \\
\text { subgroup: ananassae }\end{array}$ & 8 & $13.75(12)$ & 一 \\
\hline species: ananassae/melanogaster & 6 & $<7$ & - \\
\hline
\end{tabular}

(b) willistoni group complex: willistoni species: willistoni/willistoni species: tropicalis/willistoni complex: paulistorum species: paulistorum/willistoni species: equinoxialis/willistoni

$\begin{array}{rll}4 & 14 & \overline{0} \\ 2 & 14 & 0.413 \\ 13 & 10.53(4 \times 8,3 \times 9,10,12) & 0.524 \\ 6 & 13 \cdot 17(10,13) & 0.656\end{array}$

\section{(c) repleta group}

complex: mulleri

species: mojavensis/mojavensis species: arizonensis/mojavensis species: mulleri/mojavensis complex: hydei species: hydei/mojavensis species: eohydei/mojavensis complex: mercatorum species: mercatorum/mojavensis complex: repleta species: repleta/mojavensis

$\begin{array}{lll}12 & 14 & \overline{0.212} \\ 13 & 14 & 0 \cdot 324 \\ 11 & 12 \cdot 45(2 \times 8,9) & - \\ 23 & 12 \cdot 74(2 \times 9,3 \times 10,12) & - \\ 12 & 13 \cdot 67(10) & - \\ 9 & 13 \cdot 67(11) & - \\ 14 & 13.43(9,12,13) & \end{array}$

(d) virilis group phylad: virilis species: virilis/virilis species: americana amer./virilis phylad: montana species: montana/virilis species: borealis/virilis

$\begin{array}{rll}11 & 13.09(2 \times 9) & - \\ 15 & 11.60(5 \times 8,9,13) & 0.540 \\ 11 & 9 \cdot 27(5 \times 8,2 \times 9,3 \times 10) & 1 \cdot 230 \\ 8 & 9.75(8,4 \times 9,2 \times 10) & 1.060\end{array}$

* D values for the melanogaster subgroup are from Eisses et al. (1979), for the willistoni group from Ayala et al. (1974), for the repleta group from Zouros (1973) and for the virilis group from Throckmorton (1982). The serrata/auraria distance was calculated from the data of Triantaphyllidis et al. (1978). 
(auraria/auraria) different from the interspecific sets when they are pooled into one set. This is most likely the result of limited number of observations. The across sub-groups transplantations have produced quite different results. Two pairs of species were involved in these tests: auraria/melanogaster and ananassae/melanogaster. In both cases no vitellogenic oocytes were observed in any of the transplanted ovaries.

\section{The willistoni species group}

The evolutionary biology of the willistoni species group has been reviewed by Ayala (1975) who placed willistoni and tropicalis into one phylad, and paulistorum and equinoxialis (together with pavlovskiana) into another. This dichotomy is reflected in our data of ovary transplantations (table 1(b)). The Wilcoxon test for the paulistorum/willistoni versus willistoni/willistoni comparison is significant $(u=1.98, P=0 \cdot 024)$, and the comparison [willistoni/willistoni+tropicalis/ willistoni] versus [paulistorum/willistoni + equinoxialis/willistoni] is also significant $(u=1.69$, $P=0 \cdot 045)$.

\section{The repleta species group}

Wasserman (1982) recognized 67 species in this group, and seven more remain to be described. The described species are assigned to five subgroups, mostly on the basis of cytological information. Our study involves seven species belonging to four subgroups. The results are shown in table $1(\mathrm{c})$. As it would be expected from the close phylogenetic relationship between $D$. arizonensis and D. mojavensis, there is no trace of inhibition of oocyte development in transplantations involving these two species. However, the third species in the complex, $D$. mulleri, produced a reduced index, but the difference cannot be established statistically (the comparison [mojavensis/mojavensis + arizonensis/mojavensis] versus mulleri/mojavensis gives $u=1 \cdot 29, P=0 \cdot 1$ ). In general, there is no significant heterogeneity in the scores from interspecific transplantations within the repleta group, and even the contrast [mojavensis/mojavensis + arizonensis/mojavensis] versus all other sets combined is barely significant $(u=1 \cdot 60, P=0.055)$.

\section{The virilis species group}

In his recent review of the virilis group Throckmorton (1982) lists 11 species assigned to two "phylads", the virilis and the montana phylad. We have examined four species of these groups, as shown in table $1(\mathrm{~d})$. The intra-phylad contrast is not statistically significant (virilis/virilis versus americana/virilis, $u=1.43, P=0.076$ ), but both inter-phylad contrasts are significantly different (virilis/virilis versus montana/virilis, $u=3 \cdot 02, P=$ 0.001 ; virilis / virilis versus borealis / virilis, $u=2 \cdot 44$, $P=0.007$ ) and the [virilis/virilis + americana/ virilis] versus [montana/virilis + borealis/virilis] contrast is also significant $(u=4 \cdot 46, P<0 \cdot 001)$.

\section{Inter-group transplantations}

We performed 14 types of ovary transplantations in which donor and host species belonged to different groups. In six donor/host combinations both species belonged to the same subgenus (Sophophora or Drosophila) and in eight combinations the two species belonged to different subgenera. With two exceptions, oocytes failed to develop beyond the pre-vitellogenic stages. This result is not dependent on subgenus alliance (i.e., it does not matter whether the donor and host belong to the same or to a different subgenus of the genus Drosophila), and also is not host-dependent. The two exceptions involve the species $D$. mercatorum. Of seven $D$. mercatorum ovaries transplanted in D. virilis, one failed to develop beyond stage 7 , two developed to stage 8 , three to stage 12 and one to the final stage of 14 . Similar results were obtained when the host species was $D$. melanogaster; of eight $D$. mercatorum ovaries, three failed to develop beyond stage 7, two developed to stage 13 and three to stage 14 .

\section{DISCUSSION}

Mahowald and Kambysellis (1980) have summarized the state of knowledge regarding vitellogenesis in Drosophila. In most species examined, there appear to be three major protein components in the egg yolk, all of which are synthesized in the fat body and are immediately secreted into the haemolymph. However, the ovary itself is capable of producing all main vitellogenin polypeptides as well as absorbing them from the haemolymph of its female host (Bownes, 1982). In our experiments the native ovaries of the host flies developed normally, which means that the presence of a foreign ovary did not interfere with the initiation and continuation of vitellogenin production or with its 
assembly by the native oocytes. Thus, the failure of foreign occytes to develop must be attributed to interspecific incompatibilities interfering with the uptake process.

Our purpose was not to study the basis of these incompatibilities, but rather to examine if there was a relation between the degree of the incompatibility and the phylogenetic distance between donor and host species. The outcomes from our transplantations fall into two discrete types. (1) Complete inability of foreign oocytes to incorporate host vitellogenin (no oocyte above stage 7), and (2) ability to incorporate vitellogenin (all ovaries with MDS above stage 7).

Intergroup transplantations belong to the first category. In this category also belong transplantations between subgroups of the melanogaster species group. In this respect, these subgroups behave as belonging to different species groups. Indeed, independent evidence suggests that the evolutionary distance between the melanogaster subgroups is much larger than the distance between the phylads of the virilis group or between the subgroups of the repleta group. This can be seen, most easily, in the study of Ohnishi et al. (1983) who have produced genetic distances between species belonging to melanogaster and virilis groups from two-dimension gel electrophoresis of total body proteins. Distances between species of the melanogaster complex or between species of the montium subgroup were about $0 \cdot 20$, distances between species belonging to different complexes of the melanogaster subgroup or to different phylads of the virilis group were about $0 \cdot 35$, but the distance between the melanogaster and montium subgroups was about $0 \cdot 80$. The opposite type of observation pertains to the repleta subgroups. Here the between subgroups values of IMDS are as high as the values between complexes of the melanogaster, montium or willistoni groups.

Intragroup transplantations belong to the second type, the one where vitellogenin accumulation in the donor ovary is possible. In this category IMDS varies among donor/host combinations. The most useful observation of our study is that this variation is in broad agreement with accepted phylogenetic divisions within subgroups. This is true for the melanogaster subgroup where the index is higher for donors belonging to the melanogaster complex than for donors belonging to yakuba complex. It is also true for the willistoni and virilis groups where within-complex or within-phylad combinations give higher index values than intercomplex or inter-phylad combinations. It is not true, however, for the repleta group where the index cannot differentiate between species subgroups.

To demonstrate further the correspondence between vitellogenin accumulation and phylogenetic relationship established from biochemical divergence, we have correlated IMDS with genetic distance from allozyme studies. We were able to obtain such distances for fourteen pair-wise combinations of species, as shown in table 1 . The sources for the genetic distances are given in the footnote to the table. Several studies have reported allozyme distances between members of the $D$. melanogaster complex (Eisses et al., 1979; Gonzalez et al., 1982; Tsakas and Tsacas, 1984). The results from these studies are in general agreement. We have chosen to use the distances given by Eisses et al. (1979) because, in addition to species of the melanogaster complex, this study includes distances between species from the melanogaster and yakuba complexes. Electrophoretic distances from different studies are not strictly comparable because of variations in the technique and, mainly, because they may employ different sets of protein loci. Yet, as has been argued in other studies adopting a similar approach (e.g., Coyne and Orr, in press), this lack of uniformity is expected to increase the noise around the correlation, rather than to introduce a systematic bias causing an artefactual correlation. The correlation is shown in fig. 1. Both, the parametric $(r=-0.60, P=0.02)$ and non-parametric (Kendall's $\quad \tau=-0.43, \quad P=0.03$ ) correlation coefficients are significant (two tailed probabilities), thus reinforcing the conclusion that the ability to accumulate vitellogenin is negatively correlated with phylogenetic distance. (These tests are not strictly valid because the genetic distances of species belonging in a cluster from a species outside the cluster are the same [Felsenstein, 1985]. Taking this into account the parametric and nonparametric correlation coefficients with their probabilities become $r=-0.58, P=0.03 ; \tau=-0.45$, $P=0.01$ ).

D. mercatorum presents an exception to the rule that no vitellogenin accumulation occurs in inter-group transplantations. Ovaries of this species developed to vitellogenic stages not only in D. mojavensis (a species of the same group), but also in $D$. virilis and $D$. melanogaster (species of different groups). Srdic et al. (1978) have also noted that $D$. mercatorum ovaries developed in $D$. virilis and D. melanogaster as well as in Zaprionus vittiger, a species of a different genus. It is obvious from these results that interspecific ovary transplantations may occasionally produce results that 


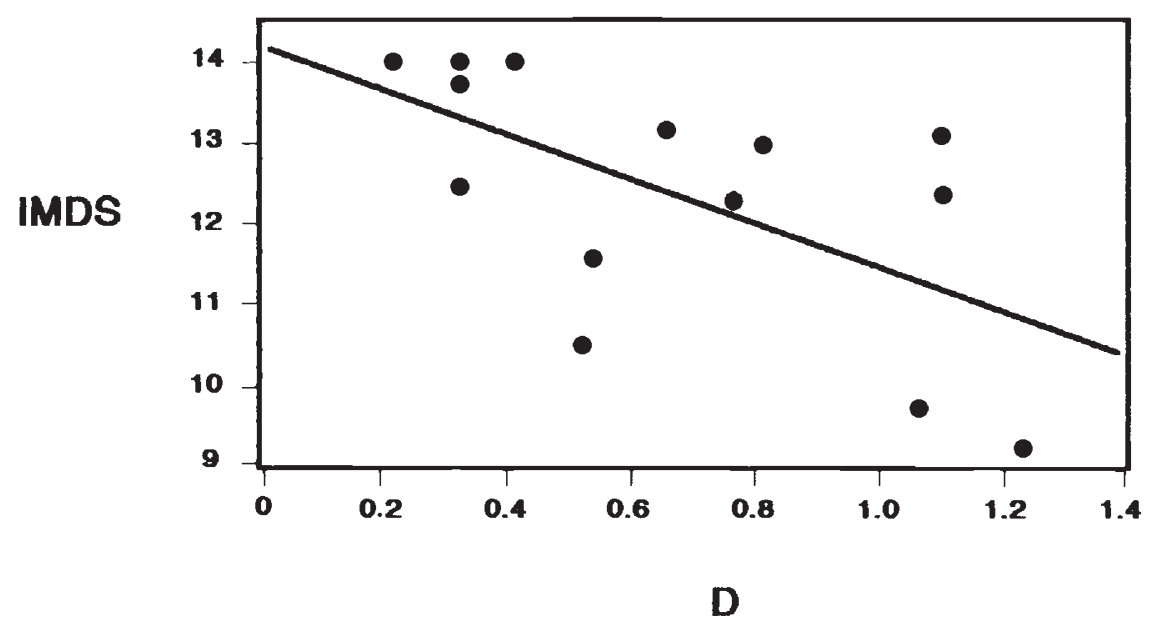

Figure 1 Plot of index of maximum developmental stage (IMDS) against allozyme distance (D) for 14 combinations of donor/host species. The species pairs and the actual values of IMDS and D are given in table 1 .

defy evolutionary relationship. The frequency and the degree of this defiance, as well as its causes, remain to be determined.

In this study we have attempted to demonstrate that interspecific transplantations can be used as an auxiliary tool for the elucidation of the evolutionary relationship between closely related species of Drosophila. Previous studies (Kambysellis, 1970; Srdic, 1978) have addressed this question at a more general level and have illustrated a broad agreement between compatibility for vitellogenin uptake and evolutionary distance. Here we examine the possibility that the degree of uptake can be used for a finer clustering of related species. One firm conclusion is that the assay has no power beyond the species-group level. The index is the same (zero) whether one compares the melanogaster group with the willistoni group or with the repleta group, even though it is known that the former two groups are phylogenetically closer. Also, the assay cannot distinguish between crosshybridizing species. The compatibility index between individuals from such species is as high as between individuals from the same species. But the assay appears to be useful for distinguishing between intermediate levels of divergence such as subspecies, species-complexes, or speciessubgroups. These levels, together with crosshybridizing complexes, present most difficulties in taxonomic studies, and also are intrinsically more interesting for the study of the dynamics of reproductive and morphological divergence.

Quite apart from the usefulness of the assay in taxonomic studies, the study has demonstrated that the functional compatibility between sets of genes separated in the course of evolution into independently evolving units (species) is related to conventional genetic distance. Clearly, a much larger set of data, preferably including many more cases of reciprocal transplantations, will be needed to establish more firmly and measure more accurately the relationship between genetic distance and vitellogenin accumulation index implied by fig. 1 . Normally, functional compatibilities (or developmental programs) can only be studied in very closely related species (as hybrid fertility, inviability or morphological abnormality). Assays that have the potential to measure these compatibilities in more distantly related species and express them in quantitative rather than qualitative terms are much in need. They alone could provide information for judging how well the rates of evolution of molecular characters, obeying a time clock [either because of neutrality (Kimura, 1983) or because of stochastic variation in selection coefficients (Gillespie, 1988)], are related to rates of divergence at loci that control various developmental processes. Our study suggests that the correlation may turn out to be good.

\footnotetext{
Acknowledgements We thank Drs F. Lemeunier, W. Heed and $Z$. Scouras for providing us with species of Drosophila, and Dr J. Szabad for technical support and advice. K.L. acknowledges financial support from the Greek Ministry of Industry, Energy and Development for a visit to Szeged, Hungary in connection with this work. This research was supported in part by an operating grant from NSERC of Canada to E.Z.
} 


\section{REFERENCES}

AYALA, F. J. 1975. Genetic differentiation during the speciation process. Evolutionary Biology, 8, 1-78.

AYALA, F. J., TRACEY, M. L., HEDGECOCK, D. AND RICHMOND, R. C. 1974. Genetic differentiation during the speciation process in Drosophila. Evolution, 28, 576-592.

BOWNES, M. 1982. Ovarian yolk-protein synthesis in Drosophila melanogaster. Insect Physiol., 28, 953-960.

BROWNLEE, K. A. 1965. Statistical Theory and Methodology in Science and Engineering, 2nd edn. Wiley, New York.

COYNE, J. A. AND KREITMAN, M. 1986. Evolutionary genetics of two sibling species, Drosophila simulans and $D$. sechellia. Evolution, 40, 673-691.

COYNE, J. A. AND ORR, H. A. 1989. Patterns of speciation in Drosophila. Evolution (In press).

EISSES, K. T., VAN DIJK, H. AND VAN DELDEN, W. 1979. Genetic differentiation within the melanogaster species group of the genus Drosophila (Sophophora). Evolution, 33, 10631068.

FELSENStE IN, J. 1985. Confidence limits on phylogenies: An approach using bootstrap. Evolution, 39, 783-791.

GILlESPIE, J. H. 1989. Molecular evolution and the neutral allele theory. Oxford Surveys Evol. Biol. (In press).

Gonzalez, A. M., CABRERA, v. M., LARRuga, J. M. AND GULLON, A. 1982. Genetic distance in the sibling species Drosophila melanogaster, Drosophila simulans and Drosophila mauritiana. Evolution, 36, 517-522.

KAMBYSELLIS, M. 1970. Compatibility in insect tissue transplantations, ovarian transplantations, and hybrid formation between Drosophila species endemic to Hawaii. J. Exp. Zool., 175, 169-180.

KING, R. C. 1970 . Ovarian Development in Drosophila melanogaster. Academic Press, New York.

KING, R. C. AND BODENSTEIN, D. 1965. The transplantation of ovaries between genetically sterile and wild type Drosophila melanogaster. Z. Naturforschung, 20, 292-297.

KIMURA, M. 1983. The Natural Theory of Molecular Evolution. Cambridge Univ. Press, Cambridge.

LEMEUNIER, F., DAVID, J. R., TSACAS, L. AND ASHBURNER, M. 1986. The melanogaster species group. In Ashburner, M., Carson, H. L. and Thompson, J. N. Jr. (eds) The Genetics and Biology of Drosophila, vol. 3e, Academic Press, London, pp. 148-239.
MAHOWALD, A. P. AND KAMBYSELLIS, M. P. 1980. Oogenesis. In Ashburner, M. and Wright, T. R. F. (eds) The Genetics and Biology of Drosophila, vol. 2d, Academic Press, London.

OHNISHI, S., KAWANISHI, M. AND WATANABE, T. K. 1983. Biochemical phylogenies of Drosophila: protein differences detected by two-dimensional electrophoresis. Genetica, 61, 55-63.

postlethwait, J. H. AND handler, A. M. 1978. Nonvitellogenic female sterile mutants and the regulation of vitellogenesis in Drosophila melanogaster. Develop. Biol., 67, 202-213.

SRDIC, Z., BECK, H. AND GLOOR, H. 1978. Yolk protein differences between species of Drosophila. Experientia, 34, 1572-1574.

THROCKMORTON, L. H. The virilis species group. In Ashburner, M., Carson, H. L. and Thompson, Jr (eds) The Genetics and Biology of Drosophila, vol. $3 \mathrm{~b}$, Academic Press, London, pp. 227-289.

TRIANTAPHYLLIDIS, C. D., PANOURGIAS, J. N. AND SCOURAS, Z. G. 1978. Isozyme variation and phylogenetic relationships among six species of the montium subgroup of the Drosophila melanogaster species group. Genetica, 48, 223227.

TSAKAS, S. C. AND TSACAS, L. 1984. A phenetic tree of eighteen species of the melanogaster group of Drosophila using allozyme data as compared with classifications based on other criteria. Genetica, 64, 139-144.

WASSERMAN, M. 1960. Cytological and phylogenetic relationships in the repleta group of the genus Drosophila. Proc. Natl Acad. Sci. USA, 46, 842-959.

WASSERMAN, M. 1982. Evolution of the repleta group. In Ashburner, M., Carson, H. L. and Thompson, J. N. Jr. (eds) The Genetics and Biology of Drosophila, vol. 3b, Academic Press, London pp. 61-133.

ZOUROS, E. 1973. Genic differentiation associated with the early stages of speciation in the mulleri subgroup of Drosophila. Evolution, 27, 601-621. 